\title{
LINKED DATA GENERATION FOR THE UNIVERSITY DATA From LEgacy DATABASE
}

\author{
Arup Sarkar ${ }^{1}$, Ujjal Marjit $^{2}$ and Utpal Biswas ${ }^{3}$ \\ ${ }^{1}$ Department of Computer Sc. \& Engineering, University of Kalyani, Kalyani(W.B.), \\ India \\ arup@klyuniv.ac.in \\ ${ }^{2}$ C.I.R.M., University of Kalyani, Kalyani (W.B.), India \\ sic@klyuniv.ac.in \\ ${ }^{3}$ Department of Computer Sc. \& Engineering, University of Kalyani, Kalyani (W.B.), \\ India \\ utpal01ineklyuniv.ac.in
}

\begin{abstract}
Web was developed to share information among the users through internet as some hyperlinked documents. If someone wants to collect some data from the web he has to search and crawl through the documents to fulfil his needs. Concept of Linked Data creates a breakthrough at this stage by enabling the links within data. So, besides the web of connected documents a new web developed both for humans and machines, i.e., the web of connected data, simply known as Linked Data Web. Since it is a very new domain, still a very few works has been done, specially the publication of legacy data within a University domain as Linked Data.

In this paper our approach concentrates on publishing the data from our University's databases as linked Data on the web and also ensuring the trustworthiness of the data by addition of provenance data along with. For this purpose we have introduced a framework to make all the steps towards the Linked Data publication easy for legacy databases within a university domain.
\end{abstract}

\section{KEYWORDS}

D2R Map, Linked Data, RDF, Semantic Web \& SPARQL

\section{INTRODUCTION}

Invention of web changed the definition of information sharing. It becomes possible to store and retrieve information on the web using documents. These documents also can be connected using hyperlinks so that one can traverse from document to document through these hyperlinks. But it does not make any sense to the machines. They don't understand what information these web documents actually hold. Among the crowd of rich syntaxes, semantics remains lost. During the past few years we have seen several evolutionary developments through the Web, which changes this picture to some extent. Semantic Web technology is unquestionably one of them. According to W3C Semantic Web activity staffs [1] "The Semantic Web provides a common framework that allows data to be shared and reused across application, enterprise, and community boundaries". This technology gets boosted with one more concept from its inventor Tim Berners-Lee. And this concept is about the linking up all the data on the Web, so that a parallel Web of Data gets evolved besides the traditional Web. This Web of Data would be completely machined processable and understandable. For Semantic Web an efficient ontology represents an important aspect. Kapoor \& et al. [2] describes different types of ontology management tools like Protege, DOI : 10.5121/ijwest.2011.2302 
International Journal of Web \& Semantic Technology (IJWesT) Vol.2, No.3, July 2011

Apollo, IsaViz, Swoop etc. They gave a comparative study of these tools in their paper [2]. At present OWL is most popular to use for ontology development, but to achieve the goal of the Linked Data RDF is enough and quite efficient for now.

According to Tim Berners-Lee's proposal [3], in Linked Data, all the resources are represented by URI. These URIs are derefferenciable using the HTTP protocol. They are searchable for some standard information for humans and machines, programs or agents by using some query language like SPARQL. This datasets may be linked with other datasets, so, they will be discoverable easily from those datasets and vice versa.

Although, a simple question still remains unanswered, "Why Linked Data is important while we have Semantic Web technology already?" Actually Semantic Web which is also known as Web of Data represents a goal, where the data on the web can be put completely machine processable. Applying Linked Data [4] concept is just a big and efficient step towards this goal which assures a possibility of a global machine processable data space.

In this paper, we have presented a framework by following the above said path trails. This framework is designed for publishing the University's legacy database as Linked data on the Web of Data .

Rest of paper is organised as follows. In section 2, a brief introduction to the related work is presented which will help the readers to understand the working domain better where as few words are expended telling about the private or public nature of Linked Data in section 3. In section 4 we discuss about the provenance of the web of data. After discussing the motivation behind the work proposed framework for Linked Data generation form legacy University database has been illustrated in section 6.In last section 7 concludes the paper.

\section{RELATED WORK-A LITERATURE REVIEW}

Semantic Web technologies can be used in many different domains. Currently e-Governance has attracted several researchers' interest. Where semantic technology can play a key role. FonouDombeu \& et al. discusses this issue in their paper[5] quite well. They discuss how the RDF, OWL and other tools like protégé or Jena API could be used to build domain ontology for the eGovernance domain. Though we did not consider the development of domain ontology, rather we concentrate our focus on the publication of data of the databases from an e-Governance domain like university as Linked data, so that it can be a part of a global data space.

If we look through the literature we can find less but some interesting work about Linked Data and its applications. One of the famous applications of Linked Data is DBpedia ${ }^{1}$. This project is dedicated to publish the structured data from Wikipedia project to the Web of Data as Linked Data. An excellent description on DBpedia is available at their website. Besides this C.Bizer \& et al. also discussed prominently about this project in [6]. In this article they describe the knowledge base extraction from the DBpedia dataset. They also discuss about the current status of DBpedia as centre of interlinked data sources through different Linked Data applications on the Web of Data.

A very interesting application based on Semantic Web technology and Linked Data principles is the Revyu [7] a wonderful piece of work done by Heath \& et al. It is a publicly available reviewing and rating Web site where we can review and rate almost anything. It is generally comes with an user friendly Web interface implemented using PHP, a very well known, easy and, lightweight but powerful server side scripting language. Revyu generally takes input from the end

${ }^{1}$ www.dbpedia.org/ 
users and generates machine readable RDF data based on it. It also minimizes the user's load by maximizing the reuse of external data sources by generating the structured data from user's simple inputs automatically and exploring the links to the external data sources automatically assigning URI to all the things at Revyu. As it is stated, the functionality of Revyu depends on user inputs, it does not consider the potential of the legacy databases holding information that may match the purpose of Revyu.

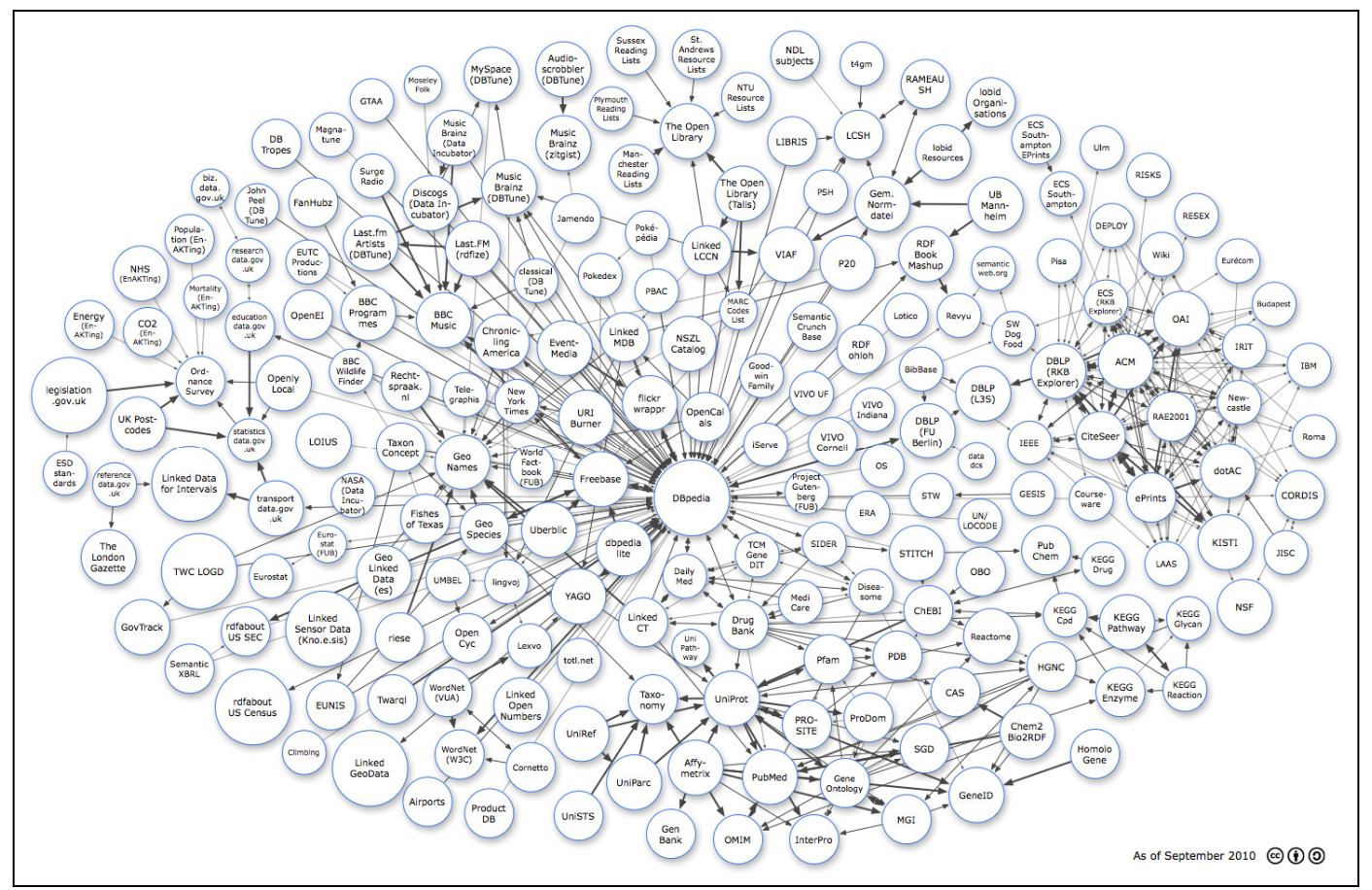

Figure 1. Linking Open Data cloud diagram, by Richard Cyganiak and Anja Jentzsch. http://lodcloud.net/

Still now the most well known project adopting the Linked Data principles is Linking Open Data community project. In the early days of the project researchers and developers are only involved but now several large organizations like BBC, Thomson Reuters etc. [8] also has joined. As of September 2010 this project holds 203 data sets consists of 25bilion RDF triples including 395 milion interlinks among them. Figure 1 represents the present LOD cloud digram. In the figure every circle or node represents a datasets and the arrows among them represents the existing interlinks between the different datasets. As shown in the figure a single data set can be interlinked with more than one other datasets. By navigating through this links on e can discover the related data items from one data set to another.

If you are crazy about movies and likes to read reviews and summary of different movies, then there is a huge possibility that you are fully aware about IMDB project where reviews and much other information about the movies of different genre are maintained. There is also an IMDB ${ }^{2}$ like project in the Web of Data is called LinkedMDB [9]. LinkedMDB publishes RDF data about movies, including interlinks to several datasets in the LOD Cloud. Linked-MDB currently contains over 3.5 million RDF triples with information about more than 100,000 movie-related entities (e.g., films, actors, characters, directors, producers, editors, script writers, music composers, soundtracks, ratings, festivals) obtained from open, non-RDF, datasets This project

\footnotetext{
${ }^{2} \mathrm{http}: / / \mathrm{www} \cdot \mathrm{imdb} . \mathrm{com} /$

${ }^{3}$ http://www.linkedmdb.org/
} 
also publishes movie reviews and other related information as Linked Data. LinkedMDB ${ }^{3}$ is also connected with many of the external data sources like DBpedia, Geonames, Musicbrainz, IMDB etc. The highlight of LinkedMDB's contribution is the large number of high quality interlinks to datasets on the LOD Cloud, as well as RDF links to related web pages. LinkedMDB uses D2R server [10] to publish information from their database as Linked Data.

A case study on Linked data generation and consumption elaborately developed and discussed in [11]. Whereas a very simple but useful introduction about the generation of rich web application by exploiting linked Data Web can be found in [12]. Payder \& et al. [13] has published a paper describing a similar work as ours. They also applied LinkedData concept to publish the data of Ferdowsi University of Mashhad. One important feature of their work is that they also published the Farsi data from their database as Linked Data.

\section{Linked Data: Private or Public}

We have spent so many words about the Linked Data, but we still did not say anything about the nature of the Linked Data, i.e. "what kind of data they are, public or private?" The answer is "both". It means Linked Data can be private as well as public. The nature of the Linked Data is totally dependent on the developers. It is important to understand this simple issue at the first space. There is a common misconception that all the data that published on the Web of Data is public in nature and open for all, especially while we are using "Linking Open data (LOD) ${ }^{4}$, project as an example of Linked Data. It is to be remembered that publishing any Linked Dataset on the Web of Data does not assure that they have become a part of LOD project. To be included in the LOD project as well as in the LOD cloud diagram every Linked Dataset has to be public and they have to follow certain rules respectively. If anyone liked to search for public or private Linked Data, online CKAN ${ }^{5}$ registry would be a good place to start. And if anyone like to know about the rules to be a part of the LOD cloud diagram the LOD cloud diagram page at Richard Cyganiak's website ${ }^{6}$ will be useful.

\section{Provenance of Linked Data}

When we are talking about provenance value of data, two questions certainly arise in our mind “i) What is provenance of data? ii) Why provenance of data?" Basically, provenance data states about the origin of the data and much more. We have already seen that semantic web is nothing but, extended version of the traditional web to make them machine processable as well as machine understandable. So, naturally this technology inherits all the pros and cons of the traditional web and, among them most concerning features are the security and the trustworthiness of the data. Web is an open place for all. Anyone can avail any kind of information and, it is very much true for the semantic web as well as the Linked Data world. Security features are still under development for the semantic web technologies and its applications and, provenance does not throw any light here. Rather, it at least ensures the trustworthiness of the available data form Linked Data to some extent. By tossing the provenance data we can assess the data quality that we are trying to find. In [14], Hartig has raised a question in favour of the provenance related metadata publication for Linked Data on Web after a discussion on the provenance data itself.

In general, Provenance is tracing the lineage of where data came from, who created it, who manipulated it, how it came into existence, where it's being stored, what is the quality of data etc. The provenance of Web of data helps users to determine who can use the Web contents or

${ }^{4}$ http://www.w3.org/wiki/SweoIG/TaskForces/CommunityProjects/Linkin gOpenData

${ }^{5}$ http://ckan.net/group/lodcloud

${ }^{6}$ http://richard.cyganiak.de/2007/10/lod/ 
International Journal of Web \& Semantic Technology (IJWesT) Vol.2, No.3, July 2011

resources, what are the access permissions, what is the quality and trustworthiness of the data, why and how the data items from different sources are linked with each other, the information about the service providers (such as D2R), data publishers, data access methods and many more. It is somehow difficult to get the provenance related information in the current exercise of Linked Data since such information is not recorded into the dataset by data providers during data creation. Integrating the provenance information into the Linked Data will bring many benefits to data consumers.

Authors in [15] has shown their work about recording the provenance data such as information about links between related data items into the Biological data-set which helps users/scientists to obtain the information about particular Biological term easily. To integrate provenance of data into Linked Data requires vocabularies such as VoID that can be used to describe metadata about datasets. In [16] authors has described about the need of provenance information into the Linked Data and have introduced a new vocabulary- voidp [17], a light-weight provenance extension for the VoID vocabulary that allows data publishers to add provenance metadata. Olaf Hartig in [14] has made several discussions about the same and proposed a provenance model that captures both information about web-based data and information about the creation of data.

\section{Motivation}

By name as well as its presence Linked Data is very new to the world. Though, some progression is already seen in a number of particular areas. But still, this new breed of Semantic Web technology is under experiment. The adoption of this potential technology is facing lack of awareness. Our aim is to introduce the Linked Data concept for our University and prepare a framework for publication of departmental data as Linked Data.

Besides this, quality of data is also the matter of importance [14]. Quality of data can be tested easily by evaluation of the provenance information. We can also be assured about the trustworthiness of the information. This issue is also motivated us to do some experiment by adding provenance data with the generated university linked data.

\section{Proposed Framework for Linked Data Generation from Relational Database}

A framework for linked data generation is depicted in the following figure2. The framework is comprised of five subsections namely 1) Legacy data zone (DB), 2) RDF dump generation module (in the figure it is shown as STAGE 1), 3) Provenance handling module (STAGE 2), 4) Linked data publishing/generating components (STAGE 3) and finally 5) Linked Data zone. The above-mentioned modules 2, 3 and 4 hold the important feature of this framework.

Section 1 preserve the legacy data in the context of our university's database holding the data related to the University of Kalyani in RDBMS form. In section 2 there is component called RDF-Dumper which has two jobs. First it will read the available data of the database from the "Legacy data zone" and will generate the mapping file in N3 format. Its second job is the generation of the RDF dump file from the map file. After that this data will be delivered to the section 3 where provenance handler will collect the dump file and will add the provenance related data to it. Then this modified dump file will be submitted to the section 4. Here the LD publisher will handle the content negotiation related problems and finally it will publish the available data from the modified dump file to the Web-of-Data as Linked Data. 
International Journal of Web \& Semantic Technology (IJWesT) Vol.2, No.3, July 2011

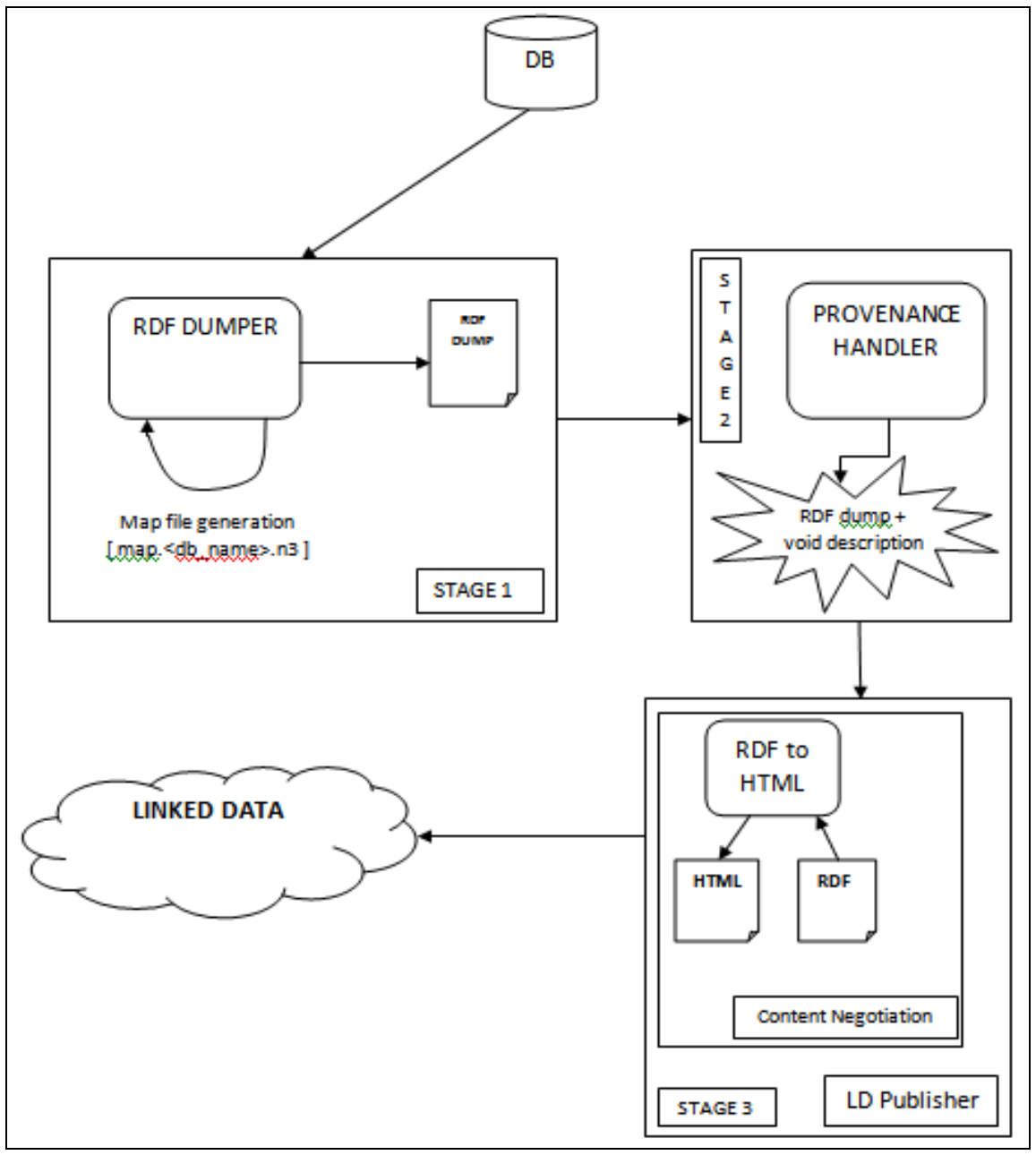

Figure 2. Proposed framework for LD generation

The detailed descriptions of each of the components are given below:

DB (University Data): it represents the legacy database. In the context of our university it is the database holding information related to the University's departments, its faculties, subjects, teachers and their student's details etc. but here during the testing phase we have developed a sample database "klyuniv" for the sake of experiments. Klyuniv have the seven tables, "departments, faculty, nonteaching_staff, publications, students, subjects and teachers". The field details of each of the database tables are given below: 
Table 1. Database structure.

\begin{tabular}{|l|l|l|}
\hline Table names & Attributes & Primary key \\
\hline DEPARTMENT & $\begin{array}{l}\text { DEPT_CODE, DEPT_NAME, } \\
\text { FACULTY }\end{array}$ & DEPT_CODE \\
\hline FACULTY & FACULTY_ID, TITLE, DEAN & FACULTY_ID \\
\hline NONTEACHING_STAFF & $\begin{array}{l}\text { STAFF_ID, FNAME, LNAME, } \\
\text { POSITION, GRADE }\end{array}$ & STAFF_ID \\
\hline PUBLICATIONS & $\begin{array}{l}\text { PAPER_ID, AUTHOR_FNAME, } \\
\text { AUTHOR_LNAME, } \\
\text { PUB_LINK, AUTHOR_TYPE, } \\
\text { AUTHOR_ID }\end{array}$ & PAPER_ID \\
\hline STUDENTS & $\begin{array}{l}\text { REGNO,SESSION,FNAME, } \\
\text { LNAME, FOAFLINK }\end{array}$ & REGNO \\
\hline SUBJECTS & $\begin{array}{l}\text { SUB_CODE, CATEGORY, } \\
\text { SUB_NAME, SYLLABUS }\end{array}$ & SUB_CODE \\
\hline TEACHERS & $\begin{array}{l}\text { TEACHERS_ID, FNAME, } \\
\text { LNAME, STATUS, } \\
\text { STATUS_TYPE, POSITION, } \\
\text { DEPT, FOAFLINK, JOIN_DT }\end{array}$ & TEACHERS_ID \\
\hline
\end{tabular}

RDF Dumper: It is the most important components considering the other parts of the framework. It is generally responsible for collecting information from the database and generating the actual mapping file in $\mathrm{n} 3$ format. It then processes that mapping file and generates the DUMP file for future use. Internally we have used D2RQ Map processor for this purpose. It is flexible to map the complex relation structure. D2R map done this without making any change to the original database schema [18]. It is one of the best tools available for publishing the data from legacy database to the world as Linked Data. At present we have used http://localhost:2020 as the base URI, but it can be changed to anything acceptable URI as required. In the following Table 2 a part of the mapping code generated from the University database is shown. In Table 3 a code snippets from the generated RDF dump is shown in N3 format.

Table 2. Code snippets from Map file.

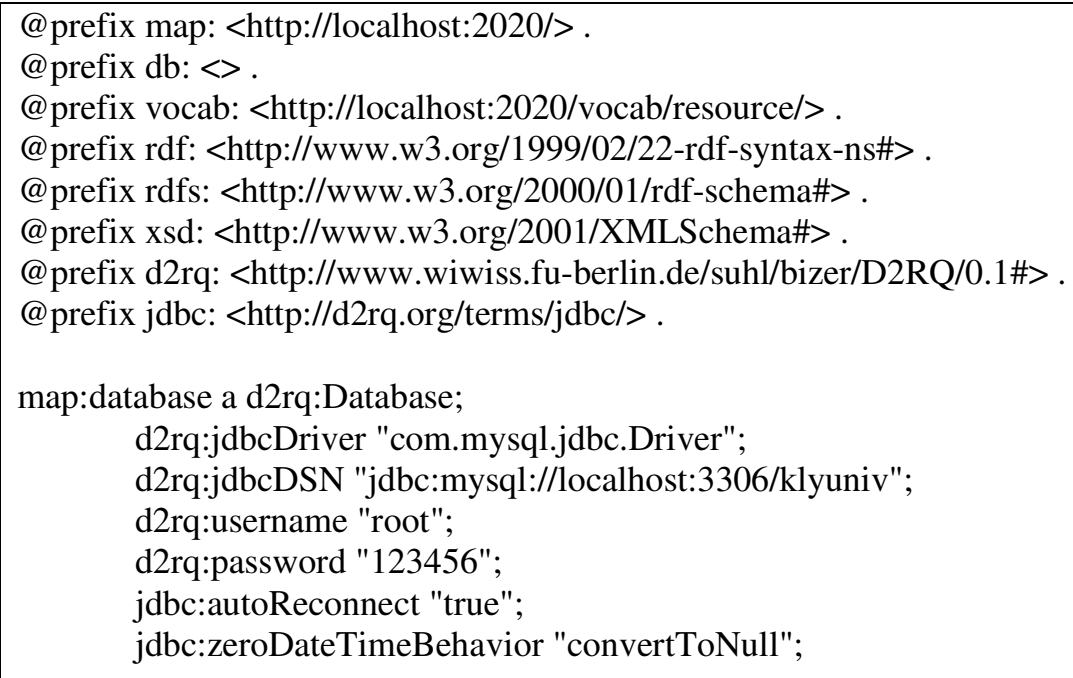


International Journal of Web \& Semantic Technology (IJWesT) Vol.2, No.3, July 2011

\# Table departments

map:departments a d2rq:ClassMap;

d2rq:dataStorage map:database;

d2rq:uriPattern "departments/@ @departments.dept_codelurlify@@";

d2rq:class vocab:departments;

d2rq:classDefinitionLabel "departments";

Table 3. Code snippets from RDF dump file.

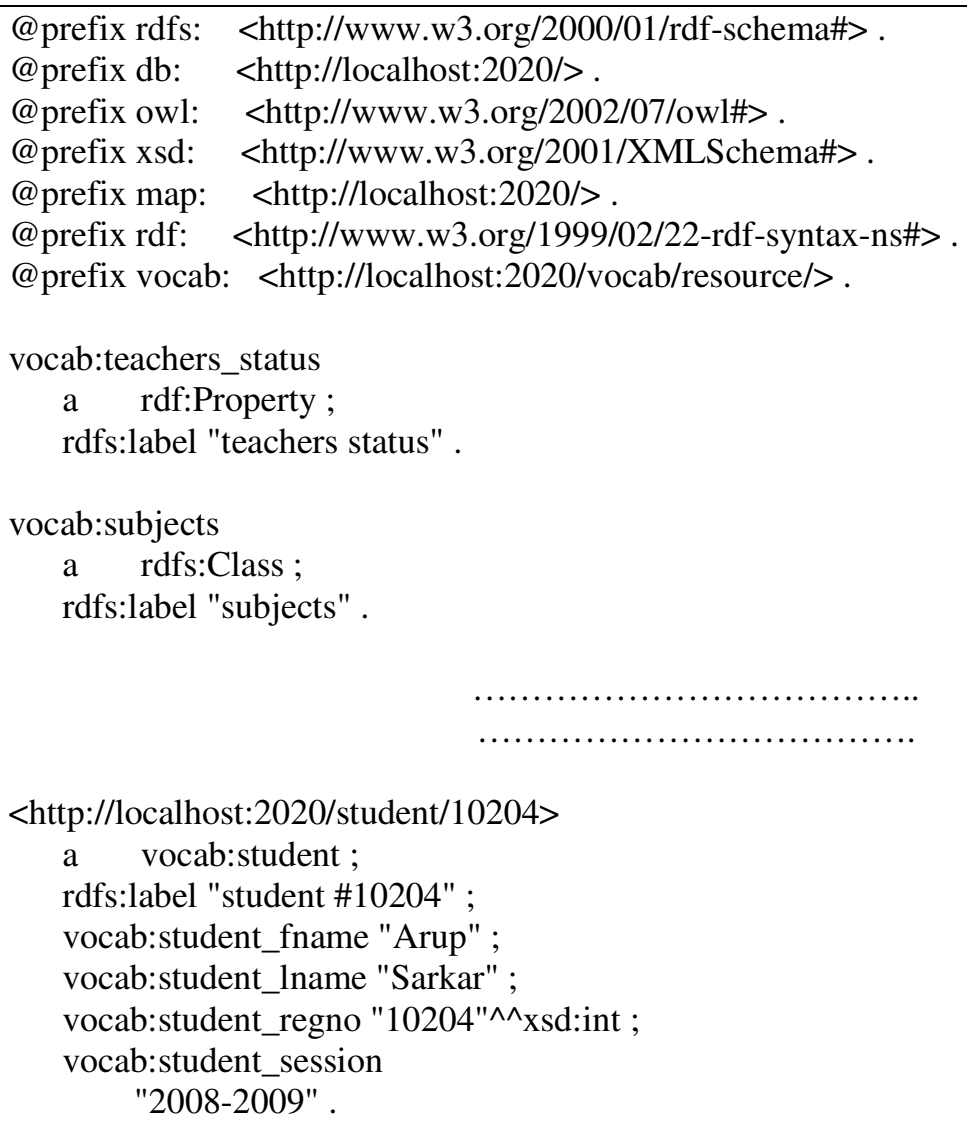

Provenance Handler: During the access of the linked data, a lot of duplicate data may be produced. So, users may face problems to choose the exact data. Provenance of the data is the main issues in this area. By studying the provenance data, decision making possibly be easy for the users to select the correct data source. Provenance Handler is totally dedicated to the 
provenance issue. Though it is fully conceptual at present. At present the addition of provenance data using the voID is added manually to generate the modified dump file. After complete development of the provenance handler tool this job can be fully automated. A sample provenance data is shown in Table 4.

LD Publisher \& RDF to HTML generator: RDF to HTML is one of important component of the LD publisher tool. It is useful while representing the RDF data as HTML for human user browsing the Linked Data. Content negotiation related issues are handled by the LD Publisher tool. At present this tools are at conceptual level and the work is in progress. We are using java servlet for these issues. Finally the Linked Data representation of our University database will be published on the web of data for consumption. To become complete Linked Data resources it must include interlinks to some related external datasets. LD Publisher generally monitors this issue internally.

Table 4. Sample provenance data for RDF dump file.

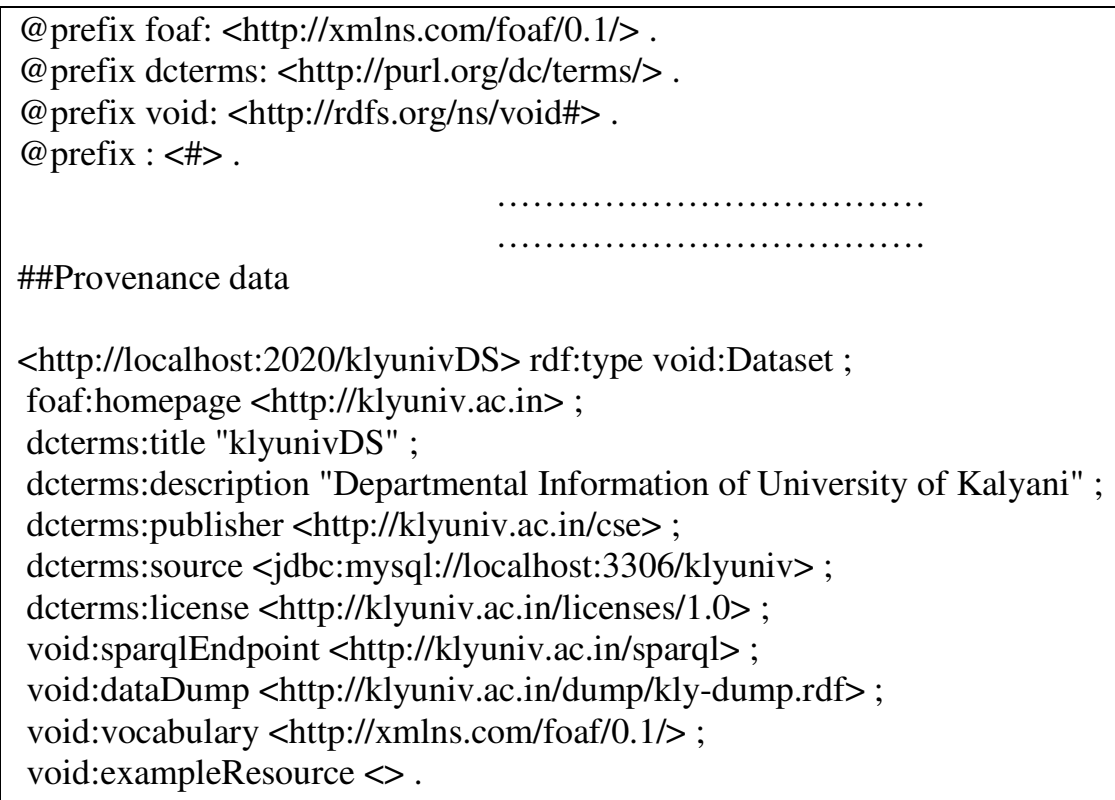

\section{Conclusions}

In this manuscript we have presented a framework which is used to publish the data from the legacy database as Linked Data on the Web of Data. In this work we have depicted the use of this framework within a University domain, but it does not limits its use for University database only. In general it could be useful for any domain, handling legacy database systems. We also represented some sample code snippets for better understanding that how structured RDF data get generated internally within the system. A sample provenance data is also shown here. Advance link discovery still at the experimental level. Interlinking with external dataset is very important for Linked Data Web. So, no doubt link discovery is very much needed for this framework. Our future work will concern this issue. Besides this, overall performance still can be improved more. We are working on it also. Though, accuracy of the mapping of data from database to RDF is not always above the question. Actually there always remains a huge possibility of inaccurate mapping or representation of data as RDF especially when these kinds of mappings are done automatically, as happened in our case. So it poses a further scope of research to improve the 
International Journal of Web \& Semantic Technology (IJWesT) Vol.2, No.3, July 2011

result. In this work we also represent a component for provenance data handling which will definitely increase the trustworthiness of the generated data. But this does not protect the data from malicious attacks. Since the openness and impact of Linked Data is much higher than its predecessor, so security issues cannot be ignored any more, and it requires a more wide research work.

\section{REFERENCES}

[1] S. Hawke, I. Herman, E. Prud'hommeaux and R. Swick, "W3C Semantic Web Activity", Available at: http://www.w3.org/2001/sw/, [Accessed on May,2011].

[2] B. Kapoor and S. Sharma, "A Comparative Study Ontology Building Tools for Semantic Web Applications," International Journal of Web \& Semantic Technology (IJWesT), Vol. 1, No. 3, pp. 1-13, July, 2010.

[3] Tim Berners-Lee, Linked Data - Design Issues, 2006, Available at http://www.w3.org/DesignIssues/LinkedData.html, Accessed on March, 2011.

[4] C. Bizer, “The Emerging Web of Linked Data”, IEEE Intelligent System, Vol. 24, No. 5, pp. 8792, October, 2009.

[5] J.V. Fonou-Dombeu and M. Huisman, "Combining Ontology Development Methodologies and Semantic Web Platforms for E-government Domain Ontology Development", International Journal of Web \& Semantic Technology (IJWesT), Vol. 2, No. 2, pp. 12-25, April, 2011.

[6] C. Bizer, J. Lehmann, G. Kobilarov, S. Auer, C. Becker, R. Cyganiak, and S. Hellmann, "DBpedia - A crystallization point for the Web of Data", presented at Journal of Web Semantics: Science, Services and Agents on the World Wide Web, Vol. 7, No. 3, pp.154-165, 15 September, 2009.

[7] T. Heath and E. Motta, "Revyu: Linking reviews and ratings into the Web of Data", Journal of Web Semantics: Science Services and Agents on the World Wide Web, 6(4), pp. 266-273.

[8] C. Bizer, T. Heath, T. Berners-Lee, "Linked Data - The Story So Far", International Journal on Semantic Web and Information Systems (IJWIS), Vol. 5, No. 3, pp. 1-22, 2009.

[9] O. Hassanzadeh, and M. Conses, "Linked Movie Data Base”, LDOW2009, Madrid, Spain, April 20, 2009.

[10] C. Bizer, and R. Cyganiak, "D2R Server- Publishing Relational Databases on the Semantic Web (Poster)", 5th International Semantic Web Conference, Athens, USA, November 2006.

[11] J. LI, and Y. ZHAO, “A Case Study on Linked Data Generation and Consumption”, LDOW2008, Beijing, China, April 22, 2008.

[12] M. Hausenblas, "Exploiting Linked Data to Build Web Applications", IEEE Internet Computing, 2009.

[13] S. Paydar, M. Kahani, B. Behkamal, M. Dadkhah, and E. Sekhavaty, "Publishing Data of Ferdowsi University of Mashhad as Linked Data", Computational Intelligence and Software Engineering (CiSE), 2010 International Conference on , pp.1-4, 10-12 Dec. 2010.

[14] O. Hartig, "Provenance Information in the Web of Data", LDOW2009, Madrid, Spain, April 20, 2009.

[15] J. Zhao, G. Klyne and D. Shotton, "Provenance and Linked Data in Biological Data Webs", LDOW2008, Beijing, China, April 22, 2008.

[16] T. Omitola, L. Zuo, C. Gutteridge, I. C. Millard, H. Glasher, N. Gibbins, and N. Shadbolt, “ Tracing the Provenance of Linked Data using VoID”, International Conference on Web Intelligence, Mining and Semantics (WIMS'11), 25-27 May, 2011.

[17] T. omitola and C. Gutteridge, "voidp: A Vocabulary for Data and Dataset Provenance”, Available at: http://www.enakting.org/provenance/voidp/, [Accessed on May, 2011]. 
[18] C. Bizer, D2R MAP - A DB to RDF Mapping Language. 12th International World Wide Web Conference, Budapest. May 2003.

\section{Authors}

Arup Sarkar is a Research Scholar of the Department of Computer Science \& Engineering, University of Kalyani, India. He obtained his Diploma, B.Tech. and M.Tech. degree in the years of 2005, 2008 and 2010 from The Calcutta Technical School, Govt. College of Engg. \& Ceramic Technology and University of Kalyani respectively. His research interests include Web Service, Semantic Web, Semantic Web Service, Ontology, Knowledge Management, Agent Oriented Programming and Web technology.

Ujjal Marjit is the System-in-Charge at the C.I.R.M.(Centre for Information Resource Management), University of Kalyani. He obtained his M.C.A. degree from Jadavpur University, India in 2000. Currently he is pursuing his Ph.D. from University of Kalyani, India. His vast areas of research interest reside in Web Service, Semantic Web, Semantic Web Service, Ontology, Knowledge Management, e-Governance as well as Software Agents etc. More than twenty two of his papers have been published in the several reputed national and international conferences and journals.

Dr. Utpal Biswas received his B.E, M.E and PhD degrees in Computer Science and Engineering from Jadavpur University, India in 1993, 2001 and 2008 respectively. He served as a faculty member in NIT, Durgapur, India in the department of Computer Science and Engineering from 1994 to 2001. Currently, he is working as an Associate Professor in the department of Computer Science and Engineering, University of Kalyani, West Bengal, India. He is a co-author of about 58 research articles in different journals, book chapters and conferences. His research interests include Optical Communications, Ad-hoc and Mobile Communications, Sensor Networks, Semantic Web Services, E-governance etc.
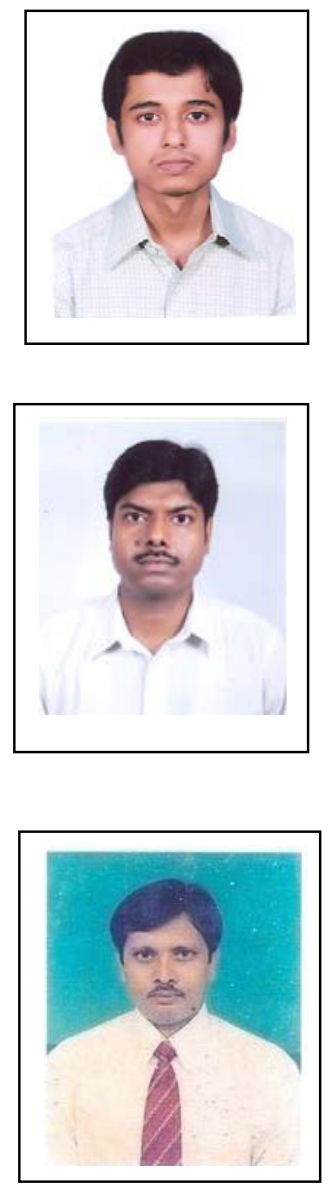